\title{
HARNESSING TECHNOLOGY TO RESPOND TO THE GLOBAL DEMAND FOR LEARNING DURING THE COVID-19 PANDEMIC
}

\author{
Richelle George ${ }^{1}$, Heini Utunen ${ }^{1}$, Christian Willems ${ }^{2}$, Ngouille Ndiaye ${ }^{1}$, Stefanie Schweiger ${ }^{2}$, \\ Thomas Staubitz ${ }^{2}$, Anna Tokar ${ }^{1}$, Melissa Attias ${ }^{1}$, Oliver Stucke ${ }^{1}$ and Gaya Gamhewage ${ }^{1}$ \\ ${ }^{I}$ World Health Organization, Avenue Appia 20, 1202 Genève, Switzerland \\ ${ }^{2}$ Hasso-Plattner-Institut für Digital Engineering gGmbH, Prof.-Dr.-Helmert-Str. 2-3, D-14482 Potsdam, Germany
}

\begin{abstract}
The COVID-19 pandemic generated an unprecedented global demand for learning about the disease and how to manage it. This paper draws on the World Health Organization (WHO)'s experience of COVID-19 knowledge-transfer to a worldwide audience of millions of learners registered on OpenWHO, WHO's massive open online course platform. It aims to illustrate the technological solutions that WHO, in collaboration with the Hasso Plattner Institute (HPI), OpenWHO's platform provider, employed in response to the unique challenges this surge in demand for learning engendered. Data on OpenWHO use, including geographic patterns and certificate attainment, were extracted from OpenWHO's internal and external reporting systems. Descriptive analysis was employed to identify trends and compare OpenWHO use with COVID-19 caseload in each WHO region. Data on the OpenWHO system load were obtained from the OpenWHO load balancer (HAProxy). The OpenWHO team responded to the need for trustworthy, evidence-based knowledge on COVID-19 via three main avenues: increased scale, targeting the needs of affected and underserved communities, and prioritising multilingualism. Each approach brought novel problems, which WHO and HPI leveraged their collaboration to meet by employing technology. This included increasing server bandwidth, expanding support teams, adding new language capabilities, and deploying functions to streamline workflows and boost learner experience. In doing so, the ability to effectively and efficiently harness technology became a critical step towards empowering learning's life-saving potential during the COVID-19 pandemic.
\end{abstract}

\section{KEYWORDS}

MOOC, e-Learning, World Health Organization, Hasso Plattner Institute, COVID-19, Technology-Enhanced Learning

\section{INTRODUCTION}

The World Health Organization Health Emergencies Programme Learning and Capacity Development (LCD) team launched the OpenWHO platform in 2017 to facilitate the transfer of public health knowledge for emergencies on a massive scale, anticipating the next pandemic. Grounded in open access and equity principles, the LCD team offered free, self-paced courses, accessible in low-bandwidth and offline formats, and available in national and local languages. While the platform served front-line responders in previous outbreaks from Ebola to plague, 2020 marked its first pandemic test.

In 2012, the year of the massive open online course (MOOC) (Pappano, 2012), the Hasso Plattner Institute (HPI), a privately financed university institute focusing on the engineering of complex information technology (IT) systems, launched openHPI, the first European platform for MOOCs. Since then, it has offered free access to current university knowledge from the rapidly changing IT and innovation fields. In that time, openHPI has registered more than 900,000 course enrolments. More than 260,000 people from 180 countries belong to the platform's permanent user base. The scalable platform infrastructure of openHPI was the basis for the cooperation with partners such as SAP (for the first Enterprise MOOC platform, openSAP) and the World Health Organization (WHO).

The LCD team began collaborating with HPI in 2016 to develop and run the online learning platform that would become OpenWHO. WHO had three requirements of the platform HPI would provide: it had to be accessible to all, have a global reach, and the ability to upscale, withstanding the traffic of millions of learners 
in the event of a worldwide health emergency such as a pandemic. An open tender process determined that HPI's technology would meet these needs, offering a highly scalable infrastructure accessible via desktop and mobile browsers (and a mobile app) and suitable to use in areas with reduced internet connectivity. HPI's approach had already proven to be successful in running MOOC platforms such as openSAP and openHPI.

The COVID-19 pandemic has altered how people conduct their lives, and the extent to which may only be apparent in years to come. There has been a shift towards e-learning and an urgent need to educate and engage health care professionals, communities and the general public (Aldohyan et al., 2019; Fernández-Díaz et al., 2020; Chick et al., 2020; Zitzmann et al., 2020). Digital learning has effectively increased public health professionals' knowledge (Brown et al., 2020; Martinengo et al., 2019; Tudor Car et al., 2019; Vaona et al., 2018), rivalling the efficacy of traditional learning methods (Tudor Car et al., 2019).

Digital approaches to learning are more intuitive and interactive, facilitating students' continuous participation (Zitzmann et al., 2020) and contributing to lifelong learning, helping learners achieve better practical outcomes due to their context-specific nature (Regmi and Jones, 2020). By allowing students to work at their own pace, digital learning is more flexible, self-directive, and inclusive. As a result, digital learning can be a means to educate the adult population that is less likely to force them to choose between their professional or familial lives and a return to education (Zitzmann et al., 2020; Martinengo et al., 2019; Regmi and Jones, 2020; Carapeto and Barros, 2019).

This paper aims to examine how technological solutions deployed by HPI enabled the OpenWHO team to meet the immense global demand for evidence-based learning on COVID-19.

\section{METHODS}

Data on historical patterns in OpenWHO use, including enrolment trends, certificate attainment and the number of COVID-19 courses and languages, were obtained from OpenWHO's in-built reporting system. A descriptive statistical analysis was conducted in Microsoft Excel to identify trends. The data reflects OpenWHO use between January 2020 and January 2021 to provide insight into the first year of pandemic response on OpenWHO.

Data on geographic trends in learner activity were retrieved from Google Analytics and consist of the cumulative number of web sessions on OpenWHO by country. As measured by Google Analytics, a web session refers to the period a user actively engages with the OpenWHO platform. The data spans from December 2019 to December 2020. The data were aggregated according to the six WHO regions (African, Americas, Eastern Mediterranean, European, South-East Asian and Western Pacific). This data was subsequently merged with a dataset covering the same period extracted from the WHO COVID-19 Dashboard (World Health Organization, 2020), which records the cumulative number of confirmed COVID-19 cases worldwide.

System load data were extracted from the OpenWHO load balancer (HAProxy). It reflects the non-negative derivation of the request counter from the load balancer. The load balancer counted all requests, and the information was collected from the counter every 10 seconds. The data were used to visualise the request rate, covering 25 February to 23 March 2020. For every pixel (longer than 60 seconds), the most significant requests per minute (rpm) was displayed.

\section{RESULTS}

The OpenWHO team responded to the significant global demand for learning about COVID-19 in three ways:

1. By rapidly scaling up the production and dissemination of COVID-19 learning materials, drawing crowds of new users to the platform

2. By serving vulnerable populations

3. By widening access to learning through offering courses in the national and local languages of communities affected by COVID-19

The technological innovation and advancements executed by the Hasso Plattner Institute were critical in enabling all three aspects. 


\subsection{Upscaling OpenWHO Platform Capacity}

OpenWHO built on the collaborative systems already in place within WHO to quickly scale up course production for the pandemic, making life-saving information from WHO experts available online at a time when lockdowns and social distancing limited the ability to unite for face-to-face learning. The first COVID-19 course launched on 26 January 2020, even before the outbreak was declared a public health emergency of international concern.

Since late January 2020, the OpenWHO team has worked with more than $50 \mathrm{WHO}$ experts to produce 25 COVID-19 courses - covering everything from infection prevention and control to risk assessment for mass gatherings - which are updated as new guidance and tools become available. As working from home increased during the pandemic, the OpenWHO team devised new protocols for remote audio-visual production, allowing courses to be launched in real-time, even as the pandemic evolved.

Mainly fuelled by the COVID-19 courses, traffic to OpenWHO grew exponentially $(+2900 \%)$ in one year of responding to the pandemic. Total enrolments increased from 160,000 in January 2020 to 4.8 million in January 2021 (Figure 1). Such a massive surge in users was also uncharted territory for the HPI team.

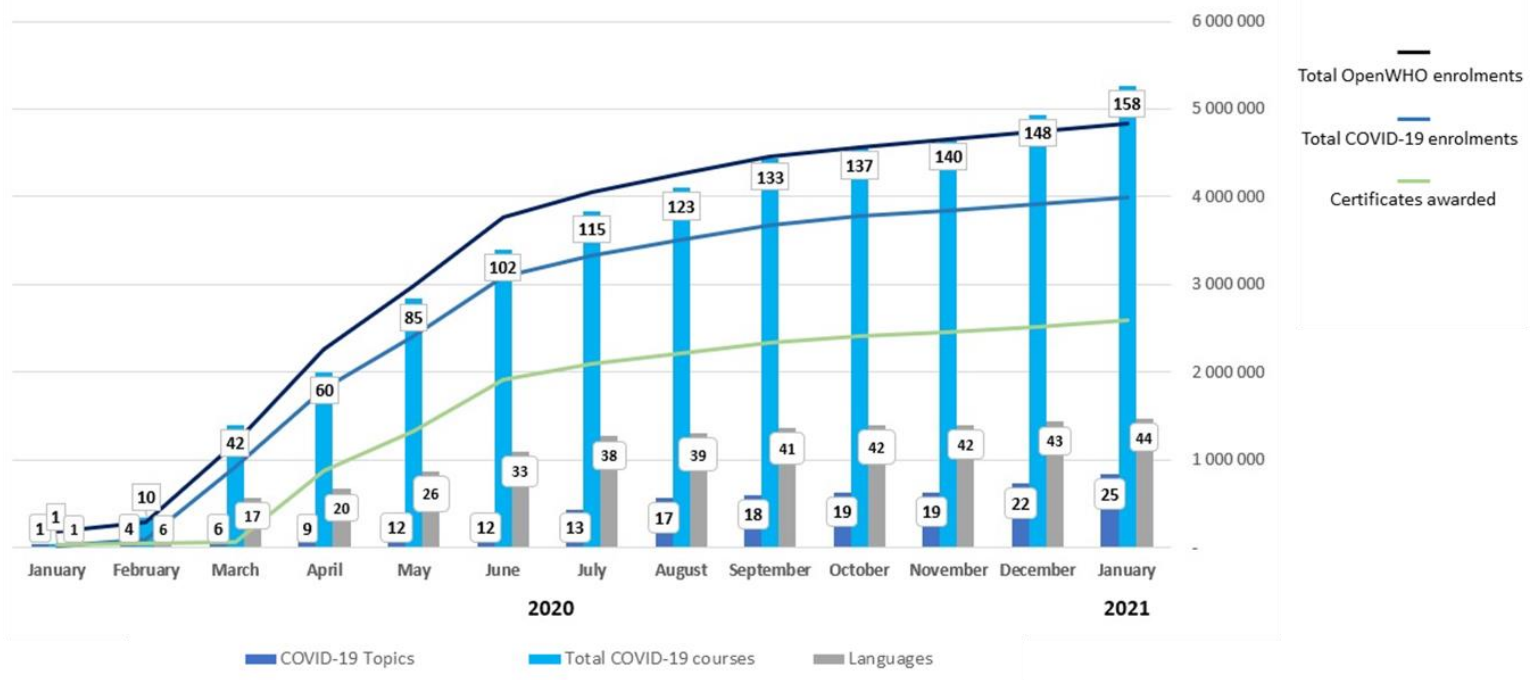

Figure 1. Growth in total OpenWHO enrolments, COVID-19 course enrolments and certificates awarded by the platform, alongside the growth in COVID-19 course and language offerings

Before it launched its first COVID-19 course, the OpenWHO platform saw a baseline load from 300 to $1,000 \mathrm{rpm}$. Between 24 January (two days before the first COVID-19 course launched) and 25 February 2020, this increased to approximately $1,000 \mathrm{rpm}$ with peaks at 2,500 rpm. 25 February was a turning point, as the number of attempts to access the platform doubled. The baseline load now lay between 2,000 and 2,500 rpm with peaks between 4,000 to 5,000 rpm. The requests increased exponentially from 15 March onwards, coming in at a minimum of 5,000 rpm, up to 15,000 and at times $20,000 \mathrm{rpm}$. Since then, the platform has witnessed peaks of well over $30,000 \mathrm{rpm}$, and 15,000 rpm during relatively idle phases, constituting a total increase in the range of 30 times the number of requests to the OpenWHO servers (Figure 2). 


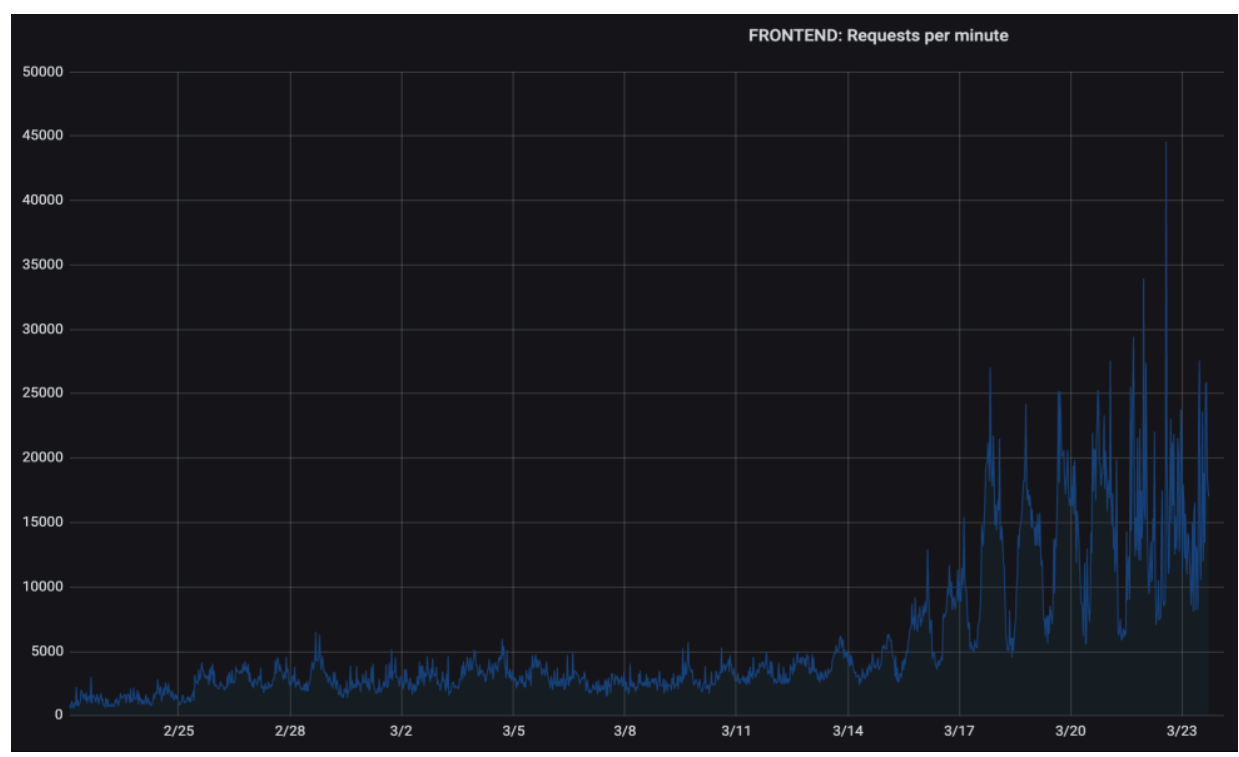

Figure 2. Requests per minute on the OpenWHO server between 25 February and 23 March 2020

The increased load led to HPI implementing several emergency measures to keep the system running stably and smoothly. The existing bandwidth of the servers' Internet connection had to be swiftly increased from 100 to $1,000 \mathrm{Mbit} / \mathrm{s}$. Due to the urgency of the situation and the pandemic's fast-evolving seriousness, HPI sidestepped the lengthy processes that would typically be involved in scheduling an appointment with a technician who would implement the bandwidth increase.

The scalable infrastructure of the OpenWHO platform was also critical in withstanding the massive change in load. Since HPI runs the OpenWHO platform in an OpenStack-based private cloud infrastructure, new virtual servers could easily be added (Figure 3).

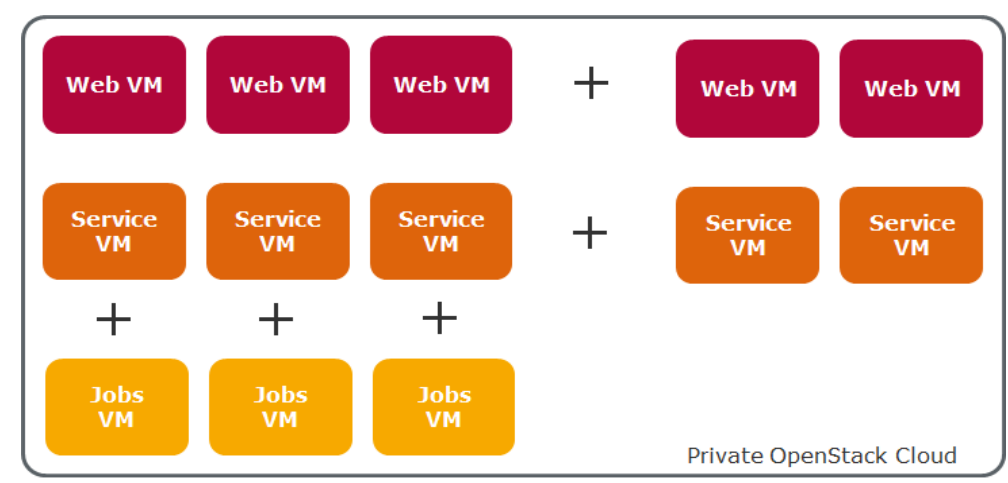

Figure 3. Scaling the OpenWHO infrastructure

Given that OpenWHO's load baseline rarely drops below 10,000 rpm, it would be safe to assume a very international audience, geographically dispersed across all time zones. This differs from other HPI-run MOOC platforms, where peak traffic times coincide with the European daytime.

\subsection{Serving the Vulnerable}

When examining OpenWHO activity in the six WHO regions, compared with the burden of COVID-19 in these regions, it is evident that OpenWHO platform use correlates with COVID-19 caseload (Figure 4). 


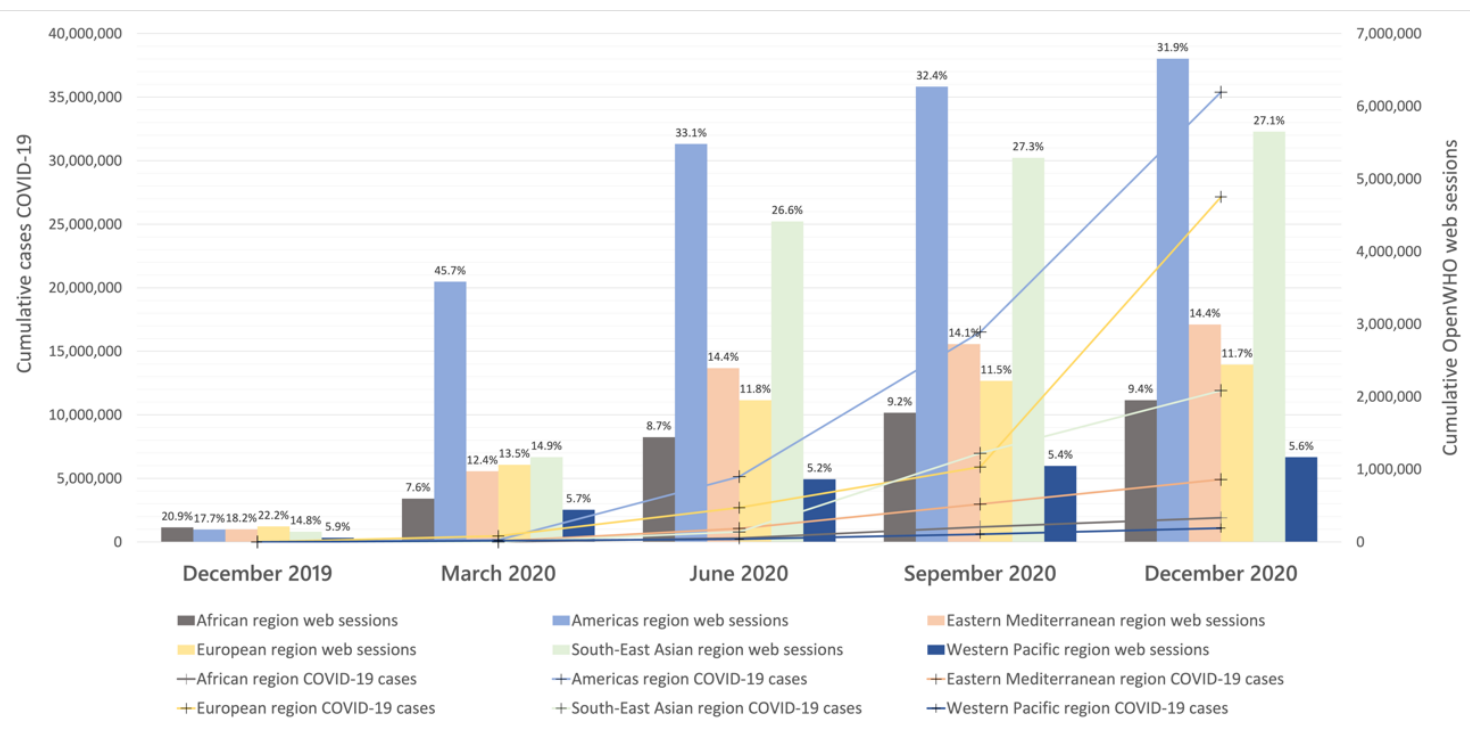

Figure 4. COVID-19 case burden and OpenWHO use by WHO region for each trimester between December 2019 and December 2020

In December 2019, the WHO African Region accounted for 20.9\% of global OpenWHO web sessions and was second only to the European Region, which made up 22.2\%. As the pandemic took hold in March 2020, the African Region's proportion fell to 7.6\%, while the Americas Region increased to $45.7 \%$ from $17.7 \%$ in the previous quarter. The Americas Region has continued to see the highest levels of OpenWHO use throughout 2020, while also being the WHO region with the highest number of COVID-19 cases in the same period. Likewise, the WHO regions of Africa and the Western-Pacific had the lowest COVID-19 caseload and were also the two regions with the least OpenWHO activity in 2020. The three other WHO regions, the Eastern Mediterranean, European and South-East Asian regions, were sandwiched in the middle, both in COVID-19 caseload and OpenWHO usage. In the final half of 2020, the break-down of OpenWHO activity among the WHO regions remained roughly static in terms of the proportion of one area to another, despite continued overall growth in the platform's use.

As the pandemic took hold, OpenWHO drew learners from an increasingly wide range of professional backgrounds and age-groups seeking knowledge about the outbreak, reflecting the far-reaching impact of COVID-19 throughout affected communities (Utunen et al., 2020). This influx of new target groups brought novel questions and problems to the platform Helpdesk, for example, regarding the anticipated availability of specific courses in new languages. The workforce required to manage the OpenWHO Helpdesk, therefore, had to increase exponentially. HPI quickly enlisted international students taking the Institute's digital health master's course to the Helpdesk support team, ensuring the team could command several languages.

HPI found a more sustainable approach to tackling the flood of requests for support via enhanced automation. They introduced the first version of a chatbot that could answer platform-specific frequently asked questions on 5 May 2020, thereby guaranteeing a 24/7 customer support source. The result was that many of the most commonly asked questions were addressed by version one of the chatbot, leading to reduced manual effort on the part of the support team and, thereby, diminished cost to HPI. On average, $40 \%$ of the users who received a chatbot suggestion did not create a Helpdesk ticket (Zobel et al., 2020).

Another feature that HPI created specifically for OpenWHO users during this time was the Certificates tab. The Certificates tab acted as a dedicated space where learners could download their course certificates. The creation of the Certificates tab not only benefited learners by making certificates easier to find and download but, in doing so, substantially reduced the certificate-related tickets submitted to the Helpdesk. Some learners contacting the Helpdesk for certificate-related support relayed that their employers and academic institutions had requested the course certificates, indicating the increasing recognition of OpenWHO courses among these groups. 


\subsection{Addressing Equity in Access to Learning through Multilingualism}

OpenWHO's COVID-19 courses are to date available in 44 languages, including the 15 most-spoken languages worldwide and languages selected specifically to reach vulnerable populations and those who lack access to trusted information about the pandemic. In support of this aim, HPI added the ability for courses to be published on the platform in 35 new languages. OpenWHO and HPI also worked together to oversee the translation and language localisation of the entire platform into the UN official languages of Chinese, French, Spanish and Russian, with Arabic under development and Portuguese also available.

The OpenWHO team has worked with translators around the world to translate roughly 7.2 million words as of January 2021. The translation process has involved harnessing the power of crowdsourcing, as the OpenWHO team partnered with WHO country and regional offices, volunteers and institutions to translate and ensure quality control. One such collaboration has been with WHO's Pan American Health Organization (PAHO). PAHO voluntarily translated OpenWHO's COVID-19 courses into Spanish and Portuguese and re-posted the courses on their learning platform, the Virtual Campus for Public Health. The re-posted courses have amassed 605,856 enrolments on PAHO's Virtual Campus for Public Health as of January 2021. In pursuit of broader linguistic representation, the OpenWHO team also initiated machine translation in 15 languages spoken by vulnerable populations.

The transcription of the audio from course videos (speech-to-text) and the subsequent translation of these transcripts remains a labour-intensive task for the OpenWHO team but ensures greater access for those with limited bandwidth and caters to learner preference. The automated transcripts sometimes require significant amounts of time to be reviewed for errors in the machine transcription. The resulting subtitles files must also be in "WebVTT" format and UTF-8 encoded, thus increasing the likelihood of human error in sharing and editing them. Translators, such as those the OpenWHO team partners with, sometimes on an ad hoc basis, are often unfamiliar with this process and its particularities. In response, HPI set out to design and develop a tool that would streamline the process by creating a single interface embedded within the OpenWHO platform. Audio from course videos would be transcribed using an automated transcription process. The resulting transcripts could be translated using automated translation and reviewed and edited by multiple administrators and translators, all within a single interface on OpenWHO. HPI students conducted an in-depth requirements analysis with HPI's main platform partners and developed a prototype. A first version of the tool will be available on OpenWHO in the coming months.

\section{DISCUSSION}

January 2021 marked one year since the launch of WHO's first online learning course on COVID-19. In that time, the OpenWHO team has significantly scaled up the production and distribution of learning on COVID-19, resulting in nearly 5 million learner registrations on the platform, concentrated mostly on the 25 COVID-19 courses it offers in 44 languages. While HPI could easily amplify the private cloud-based server infrastructure running OpenWHO to meet this massive increase in demand for learning, the server bandwidth turned out to be a bottleneck. It ultimately required a tenfold increase in the Internet connection to run smoothly.

As the pandemic progressed, the world's hardest-hit regions drew heavily on OpenWHO resources. The correlation found between OpenWHO use and COVID-19 caseload by WHO region between December 2019 and December 2020 is consistent with previous findings which illustrated that, in most cases, levels of OpenWHO use and COVID-19 caseload were similar in magnitude in the countries with the highest number of COVID-19 cases between March and May 2020 (Utunen et al., 2020). The influx of these new users increased the platform's cultural, linguistic and professional diversity and coincided with a flood of Helpdesk tickets requesting support with various platform features. To respond, HPI expanded the Helpdesk support team and initiated a chatbot that could promptly answer the most frequently asked questions. Through feedback from the Helpdesk and OpenWHO team, HPI also identified causes of suboptimal user experience and engineered tailor-made enhancements to the user interface, such as the Certificates tab.

The WHO South-East Asian Region is somewhat of an exception to the correlation witnessed between OpenWHO use and COVID-19 caseload. However, India, which makes up 78.5\% of OpenWHO learner activity in the South-East Asian Region, already dominated global MOOC enrolment numbers outside of OpenWHO before the pandemic, second only to the United States of America (Chauhan, 2017). The popularity 
of MOOCs in India might explain, in part, the way that the country has fuelled OpenWHO use in the WHO region of South-East Asia, to which it belongs. Additionally, the popularity of OpenWHO's courses in the South-East Asian and Americas regions may be somewhat explained by the availability of all OpenWHO's COVID-19 courses in English, a language widely spoken in both regions. In contrast, COVID-19 courses have been most commonly translated into Spanish, Portuguese and French - 10, 9 and 8 out of OpenWHO's 25 COVID-19 courses have been translated into these languages, respectively. OpenWHO courses are also almost always available in English before being followed by translations, giving additional time for enrolments to accumulate on English courses compared with translated ones.

The OpenWHO team sought to bolster multilingualism on the platform and partnered with translators worldwide to widen access to learning. The partnership forged between PAHO's Virtual Campus for Public Health and OpenWHO helped broaden the audience reached by the courses, particularly in the Americas Region, and illustrated the powerful potential of joint efforts between existing learning platforms during health emergency response. To support the multilingualism efforts, HPI added the functionality for 35 new course languages on the platform and began creating an automated transcript and translation tool for integration directly onto OpenWHO.

As the pandemic has evidenced, correctly and swiftly utilising technology in response to public health challenges ensures that the following critical components of online learning delivery can be satisfied:

1. Equity: OpenWHO's MOOCs are available to all at no cost, democratising knowledge and learning. Providing equitable access to health emergency knowledge in affected populations' mother tongues must remain a core value of online learning for public health emergencies.

2. Accessibility: Low-bandwidth compatible online learning (with the ability for offline-modes) can enable participants with limited technology or internet connectivity to access learning. Online learning can be cost-effective and time and resource efficient. Self-paced, low-bandwidth-adjusted, downloadable, and portable learning (available on any device and offline) can also increase knowledge accessibility.

3. Flexibility: Self-paced learning can enable individuals to progress according to their preferred speed, time, and place, catering to the learner's needs and availability.

4. Learner-centricity: User-friendly options allow individuals to choose formats specific to their learning needs and provide the basis for a more customised learning experience. In this vein, they can attract repeat learners and encourage continuous, lifelong learning.

5. Quality: Courses based on reliable and trustworthy technical guidance and adult learning techniques can produce the highest quality of content and enhance learning.

\section{CONCLUSIONS}

By quickly employing innovative and novel solutions to technological limitations, and firmly rooted in the value of equity of access, HPI and the OpenWHO team were able to work together to meet the unprecedented global demand for learning during the COVID-19 pandemic. In doing so, they were able to contribute towards the WHO's goal of reducing transmission, saving lives and protecting the vulnerable. Despite the challenges involved, the COVID-19 pandemic has given rise to the first time in its history that WHO launched fast, high-quality, accessible learning on a massive scale, with an emphasis on multilingualism.

Our study is limited by the descriptive nature of the analysis performed; further research should employ statistical tests to ascertain measures of association and statistical significance. However, our study is strengthened by the fact that it uses the complete datasets available (which record all OpenWHO web sessions and COVID-19 cases that occurred during the study period) to conduct the analysis, instead of relying on an extracted sample from the data which may be unrepresentative. Our study illustrates the challenges faced in conducting a global, multilingual, "just-in-time" online learning response to a pandemic and exemplifies technological solutions that can be rapidly and effectively leveraged to address them.

The COVID-19 pandemic has proven online learning's power to ensure equitable access to knowledge for all learners. Online learning can no longer be considered a temporary solution when in-person gatherings are impossible. Instead, it demands to be seriously considered a modern approach to efficient and impactful public health work, requiring skilful and creative use of technological solutions. It is a fundamental part of the ecosystem needed to create a culture of lifelong learning in health. 


\section{REFERENCES}

Aldohyan M., et al., 2019. The perceived effectiveness of MERS-CoV educational programs and knowledge transfer among primary healthcare workers: a cross-sectional survey. BMC Infectious Diseases, 19, 1, p. 273.

Brown A., et al., 2020. Effectiveness of technology-enabled knowledge translation strategies in improving the use of research in public health: Systematic review. Journal of Medical Internet Research, 22, 7, p.e17274.

Carapeto, C. and Barros, D.M.V., 2019. Nutrition and health as virtual class at Open University (Portugal): pedagogical strategies for higher education. International Journal of Educational Technology in Higher Education, 16, 1, p. 19.

Chauhan, J., 2017. An Overview of MOOC in India. International Journal of Computer Trends and Technology, 49, 2, pp. 111-120.

Chick C.R., et al., 2020. Using Technology to Maintain the Education of Residents During the COVID-19 Pandemic. Journal of Surgical Education, 77, 4, pp. 729-732.

Fernández-Díaz, E., et al., 2020. Exploring WHO communication during the COVID-19 pandemic through the WHO website based on W3C guidelines: Accessible for all? International Journal of Environmental Research and Public Health, 17, 16, p. 5663.

Martinengo, L., et al., 2019. Digital health professions education on chronic wound management: A systematic review. International Journal of Nursing Studies, 104, p. 103512.

Pappano, L., 2012. The Year of the MOOC, in New York Times. Accessible at <https://www.nytimes.com/2012/11/04/education/edlife/massive-open-online-courses-are-multiplying-at-arapid-pace.html>.

Regmi, K. and Jones, L., 2020. A systematic review of the factors - enablers and barriers - affecting e-learning in health sciences education. BMC Medical Education, 20, 1, p. 91.

Tudor Car L., et al., 2019. Health professions digital education on clinical practice guidelines: A systematic review by Digital Health Education collaboration. BMC Medicine, 17, 1, pp. 139-16

Utunen H., et al., 2020. Global Reach of an Online COVID-19 Course in Multiple Languages on OpenWHO in the First Quarter of 2020: Analysis of Platform Use Data. Journal of Medical Internet Research, 22, 4, p.e19076.

Utunen H., et al., 2020. Responding to Global Learning Needs during a Pandemic: An Analysis of the Trends in Platform Use and Incidence of COVID-19. Education Sciences, 10, 11, p. 345.

Vaona A., et al., 2018. E-learning for health professionals. Cochrane Database of Systematic Reviews, 1, 1, CD011736.

World Health Organization, 2020. WHO Coronavirus Disease (COVID-19) Dashboard. Accessible at <https://covid19.who.int/>.

Zitzmann N.U., et al., 2020. Digital undergraduate education in dentistry: A systematic review. International Journal of Environmental Research and Public Health, 17, 9, p. 3269.

Zobel T., et al., 2020. Improving the Scalability of MOOC Platforms with Automated, Dialogue-based Systems. IEEE Learning With MOOCs. Antigua, Guatemala, pp. 42-46. 